\title{
En la cocina de las políticas de igualdad: ¿qué ingredientes agregar a las nuevas recetas?
}

\author{
CARME AdÁN \\ Secretaría General de Igualdad \\ Xunta de Galicia \\ amorodo@wanadoo.es
}

Recibido: 07.02.2008

Aceptado: 16.06 .2008

\begin{abstract}
Porque la clave de las feministas es que el compromiso en primer término es con nosotras, con las mujeres (Marcela Lagarde)
\end{abstract}

El objetivo de la igualdad va mucho más allá de la agregación de mujeres a los espacios de poder y toma de decisiones, y ello es así porque pretende modificar toda la desigualdad de género que estructura nuestra sociedad. El proyecto es absolutamente radical y significa, tomando prestadas las palabras de Amorós, rehacer completamente la receta (Amorós, 2000,12). Una receta que, gracias al impulso de las políticas públicas de igualdad, se elabora con nuevos ingredientes con la finalidad de conseguir modificar efectivamente las relaciones entre hombres y mujeres. El mainstreaming de género se ha convertido en la estrategia más transformadora de las políticas públicas de igualdad actuales. El papel que el mainstreaming ha tomado en los últimos quince años viene determinado en buena medida por las limitaciones mostradas por las políticas de igualdad de oportunidades y de acción positiva.

Este artículo analiza la evolución de las políticas de igualdad a la luz de las conclusiones que podemos extraer del debate epistemológico feminista. Es cierto que en la historia de la teoría feminista la preocupación epistemológica se ha dado de forma periférica, se ha desarrollado como una reflexión de segundo orden que ha venido a fortalecer parte del conocimiento feminista, dejando de lado su posible dimensión política. Tanto es así que la cuestión sobre una posible vinculación entre la epistemología feminista y las políticas de igualdad debemos rastrearla fundamentalmente en textos sobre cuestiones como los indicadores o los informes de impacto de género. El resultado es que, hasta el momento, el 
abordaje de la temática epistemológica es tangencial en el diseño de las políticas de igualdad y su aplicación a las instituciones.

Para poder comprender las políticas de igualdad en el presente es clave el cómo y el desde dónde creamos conocimientos como feministas. La comunidad epistémica feminista debe plantearse estudios, indicadores y estadísticas capaces de trabajar en el objetivo compartido de mejorar la calidad de vida de las mujeres y de eliminar las estructuras que reproducen las desigualdades de género. De hecho uno de los grandes problemas para evaluar el progreso de las mujeres después de la conferencia de Beijing es disponer de los mecanismos de medida adecuados (Walby, 2005b) para tal fin. En este sentido la reflexión sobre los indicadores de género y su relación con la estrategia de mainstreaming obliga a plantearnos qué posibilidad tiene la teoría feminista para producir representaciones del mundo generizadas. Recordemos que en gran medida las nuevas interpretaciones y construcciones del feminismo han hecho uso de las propias experiencias de las mujeres como elemento constituyente del nuevo conocimiento.

En este artículo nos planteamos la necesidad de repasar desde el prisma epistemológico nociones - como género, experiencia de las mujeres y situaciónque son determinantes para comprender la evolución de las políticas de igualdad en los últimos veinte años así como para poder reflexionar sobre las ventajas y los inconvenientes que un instrumento como el mainstreaming puede conllevar.

\section{INGREDIENTES PARA UNA NUEVA RECETA: EL CONCEPTO DE GÉNERO}

Un debate actual y recurrente en la historia del pensamiento ha sido el referido al peso que toman los elementos naturales y los culturales en la explicación del ser humano y sus manifestaciones sociales. Esta polémica entre el naturalismo y el constructivismo se ha proyectado con especial importancia en lo referido a ese grupo de humanos cuyo sexo determina en buena medida su lugar en la sociedad, las mujeres. Es cierto que la división entre los sexos recorre toda la historia, pero en la modernidad adquiere un carácter significativo en la propia construcción del sujeto político. Reduciendo algunos matices, se pueden plantear dos posturas fundamentales a este respecto: por un lado, la que acentúa el carácter corporal y biológico de las mujeres, negándoles el derecho de ciudadanía, y, por otro lado, la representada por quienes problematizan la diferencia de los sexos y se cuestionan la jerarquización que implica, apoyándose en el papel de la educación y las costumbres en la formación de las mujeres.

Siguiendo esta segunda línea argumental, en los inicios del feminismo ilustrado, bien como movimiento político, bien como discurso filosófico crítico de la cultura, existe una voluntad clara por revisar qué es social y qué es natural en la configuración de lo masculino y lo femenino y las relaciones que se establecen entre estos términos. Asumir el papel de la cultura en la diferenciación de los sexos permite alejarse del determinismo natural — que las ciencias de la vida 
transformaron en determinismo biológico_- La naturalización de las mujeres marcó la mayor parte de los discursos y prácticas de las ciencias de la vida que consolidarían su poder normativo durante el siglo XIX. Por lo tanto, se puede afirmar que buena parte de las teorizaciones feministas suponen un esfuerzo por buscar herramientas analíticas que ayuden en la tarea de desnaturalizar a las mujeres. Ésta es una de las premisas que movió a Simone de Beauvoir a dedicar parte de sus reflexiones a la propia existencia de las mujeres. La pionera del feminismo contemporáneo se asombra ante la existencia de un grupo que no es grupo, de unos sujetos que no son sujetos; en resumen, de unos cuerpos sexuados en femenino que comparten el destino de ser el sexo, el otro. El segundo sexo es una obra de análisis sobre esa extraña relación que vincula a las mujeres con el sexo — como si los varones no tuviesen sexo-y las define históricamente como las diferentes, las Otras.

En ese extenso texto se establecen definitivamente las bases conceptuales de uno de los problemas fundamentales del feminismo, a saber, la reflexión en torno a qué es biológico y qué es cultural en la situación de inferioridad que padecen las mujeres. La toma de partido es clara y sin fisuras: son los elementos culturales y la educación, como piezas claves en la configuración de la experiencia vivida, y no ningún tipo de esencialismo biologicista, los que determinan el papel adjudicado a las mujeres.

Esta apuesta queda plasmada en el inicio del segundo volumen de El segundo sexo con la famosa sentencia «no se nace mujer: se llega a serlo» (Beauvoir, 1999, 13). Durante más de cincuenta años este lema ha ido socavando poco a poco como un topo los enfoques esencialistas que consideran la opresión de la mujer como un hecho natural. Al mismo tiempo que este sinfín de galerías y pasos subterráneos acababa con la tierra firme del naturalismo, entreabría la posibilidad de concebir el ser una mujer como el resultado de un complejo proceso de socialización.

Los feminismos, tanto los movimientos de mujeres como la teoría feminista, desarrollados a partir de los años sesenta, siguieron en una gran mayoría el dictum de Beauvoir y se aliaron con un constructivismo social que les permitió afrontar analíticamente el problema de la diferencia y huir de una esencia dada en el hecho de ser mujer. Sin embargo, aún quedaba una larga tarea por realizar, es decir, era necesario buscar los recursos conceptuales para enfrentarse a toda una plétora de discursos bien asentados en campos como la antropología, la sociología, la biología, la literatura, las ciencias médicas... Así, no es extraño que una de las construcciones teóricas con mayor acogida y repercusión posterior procediese del trabajo antropológico de Gayle Rubin a mediados de los años setenta (1975). Esta autora acuña la expresión sistema de sexo/género, con un gran calado en la teoría feminista del mundo académico anglosajón, para dar cuenta de todo el entramado anatómico-cultural que se esconde en las relaciones sociales de dominación.

El género se define como el conjunto de elementos culturales que, diseñados en un momento socio-histórico concreto, se inscriben en el sexo. Esta categoría, 
empleada hábilmente por las feministas, fue no sólo el instrumento de revisión de todas las áreas del conocimiento sino que consiguió entrar de lleno en la agenda política. Lo cierto es que en la actualidad el término género inunda la literatura sobre políticas públicas de igualdad. Se ha convertido en un concepto tan versátil y plástico que resulta necesario analizar su capacidad transformadora.

La historia de este concepto y toda la literatura sobre su funcionalidad es extensa y bien conocida, por lo que no consideramos de especial relevancia detenernos en la misma. Sí que entendemos que es necesario destacar su carácter relacional. De ahí que cuando hablamos de igualdad de género o de mainstreaming de género, como veremos en este artículo, si no disponemos de un marco interpretativo común podemos estar hablando de cosas en principio diferentes.

\section{2. ¿Y SI AGREGAMOS LA EXPERIENCIA DE LAS MUJERES?}

El carácter de función le ha dado al concepto de género toda su capacidad para deconstruir y desarticular discursos y políticas, pero le dificulta construir nuevos conocimiento sin contar con la vida real de las mujeres que sufren la desigualdad.

Por este motivo consideramos interesante reflexionar sobre el papel que cumplen los conceptos de género y de experiencia de las mujeres en la teoría feminista, en concreto en la epistemología feminista. Bajo este epígrafe se aglutinan las investigaciones y propuestas que en diálogo con la tradición filosófica y sociológica sobre la ciencia abordan los problemas clásicos como la racionalidad, la evidencia, la objetividad, el sujeto cognoscente, el realismo o la verdad y, al tiempo, utilizan la categoría analítica de género para articular una nueva forma de encarar estos temas. El nutrido grupo de textos y autoras que se agrupan bajo el paraguas de epistemología feminista tienen en común poner en tela de juicío la formulación básica del conocimiento científico, cuestionando la noción de ciencia en tanto que caracterizada como una visión de la naturaleza objetiva y libre de valores y de connotaciones culturales.

Con esta etiqueta nos estamos refiriendo fundamentalmente a las autoras que integran las tres grandes corrientes que ha descrito Sandra Harding (1986), a saber, empirismo feminista, teoría del punto de vista feminista y posmodernidad feminista. Con matizaciones estas grandes divisiones mantienen su utilidad para encuadrar a autoras como Helen Longino, Evelyn Fox Keller, Sandra Hartsock, Dona Haraway o la propia Sandra Harding.

El empirismo feminista tiene su origen en las investigaciones feministas referidas al ámbito de la biología y las ciencias de la vida. Los primeros análisis que se realizaron en esta línea interpretaron los sesgos androcéntricos de la ciencia como falta de cuidado y rigor en la aplicación del método científico. Las autoras embarcadas en esta tarea entendían su trabajo como una labor de denuncia de la mala ciencia. Este período suele denominarse empirismo espontáneo y sentó las bases para un segundo momento, el empirismo feminista fi- 
losófico, el cual, partiendo del presupuesto de la imposible neutralidad valorativa de la ciencia —incluida aquélla que se denominaba buena ciencia- somete a revisión el marco conceptual y los conceptos clave del empirismo. Como representantes del empirismo feminista filosófico podemos destacar a Helen Longino y Lynn H. Nelson.

La teoría del punto de vista feminista no se ha centrado exclusivamente en el problema del conocimiento y sus implicaciones sino que ha significado para el feminismo toda una revolución metodológica así como una determinada forma de orientar las investigaciones feministas exportable a muy variadas disciplinas. Esta concepción fue inicialmente formulada en el contexto de la tradición marxista. Las autoras que defienden esta posición afirman que en cualquier período histórico la visión del mundo hegemónica refleja los intereses y valores del grupo dominante. Las mujeres, como grupo excluido de los circuitos de poder, detentan una posición social especial que les proporciona un punto de vista epistemológico privilegiado, una visión del mundo menos distorsionada que la impuesta por los varones blancos de clase media. De este modo, se puede afirmar que la teoría del punto de vista se asienta en dos pilares básicos: a) todo conocimiento está situado, pues es una construcción práctica y social, b) una localización en especial, la de las mujeres, es más fiable porque proporciona un punto privilegiado para revelar algún tipo de verdad. Feministas tan notables como Nancy Hartsock, Dorothy Smith, Sandra Harding o Evelyn Fox Keller han desarrollado sus propuestas en la órbita de esta corriente.

El tercer apartado de esta clasificación se conoce habitualmente como posmodernidad feminista. Este enfoque tendría como denominador común, en opinión de Harding, negar los supuestos de las dos corrientes anteriores y añadir al análisis del conocimiento otras variables además del género, con el objetivo de enfatizar las identidades fragmentadas de la cultura actual. Sin embargo, dada la proliferación de términos que pretenden dar cuenta de estas múltiples variables que intervienen en los procesos de configuración de las identidades, como por ejemplo feminismos polifónicos, feminismo global o feminismos multiculturales, consideramos que la expresión feminismos polimorfos refleja mejor las muchas caras con las que debe arroparse una teoría que pretende interpretar un mundo estructurado sobre lo local/global al tiempo que evita la equívoca denominación de posmodernidad para los temas epistemológicos. La idea rectora de estos desarrollos reside en mantener la necesidad de que los feminismos, como teoría y práctica, no reproduzcan las formas de los discursos hegemónicos patriarcales que tendieron a la uniformización y homogenización de las realidades de las mujeres. Las representantes más destacadas son Susan Hekman y Donna Haraway.

Todas estas pensadoras destacan el papel del género en la construcción del conocimiento porque las mujeres han estado siempre presentes en su gestación (Rose, 1994), pero amén del necesario reconocimiento histórico, la posición actual de las mujeres debe aportar una nueva perspectiva. Su aportación se centra en destacar la situación de la mirada de las mujeres. Una mirada que encuentra 
en una noción clásica del feminismo su referente, la experiencia de las mujeres. Este concepto puede contribuir a comprender las aportaciones epistemológicas de la teoría feminista y a distanciarse de los meros análisis descriptivistas del género. Así, utilizando la terminología propia de la lógica formal, el género se puede definir como una función que permite a la teoría feminista comprender la experiencia de las mujeres, pero que al mismo tiempo toma su valor cuando se instancia por medio de unas variables concretas. El género es una relación que despide un valor que se percibe en forma de experiencia. Esta experiencia depende de dos narrativas. La narratividad subjetiva de las mujeres que cuentan su proceso vital, y otra que se corresponde a la que elabora la teoría feminista cuando establece los recursos analíticos para configurar esta función. Resulta obvio que las relaciones que existen entre lo masculino y lo femenino no son una invención de la teoría feminista, que sí que ha aportado los elementos teóricos que hacen las relaciones comprensibles y cambiables. De hecho el género es tan importante porque ha permitido comprender la experiencia de ser una mujer sin que ello tenga que ser identificado con el sexo sino con el valor que se desprende de la aplicación de una función a diferentes elementos culturales.

La noción de experiencia de las mujeres ha permitido valorar el trabajo no remunerado del cuidado o poner dentro de la agenda política temas relegados al ámbito de lo privado. Ha contribuido a encarnar la mirada del sujeto cognoscente pero también del propio sujeto político mujeres. Sin embargo, es un concepto que siempre tiene una cierta ligadura con un esencialismo latente, aunque sea cultural. Por este motivo es tan interesante la propuesta de la pensadora Donna Haraway, porque elimina cualquier forma de esencialismo en la construcción del sujeto mujeres y del conocimiento.

Haraway es una de las epistemólogas feministas que más ha apostado por reivindicar el papel social y de construcción del conocimiento. Sus tesis a la vez sugerentes e incisivas han sido un referente para la constitución del ciberfeminismo y, por otro lado, han contribuido a fortalecer la reflexión feminista sobre qué es ser mujeres en el cambio de siglo. Su formación científica y su contacto con los movimientos sociales la han ayudado enormemente a desarrollar un pensamiento poliédrico. Jugando siempre con la metáfora de la visión como situación - el ojo siempre está encarnado en la materialidad del cuerpo-, Haraway pretende indagar en la producción de significados que necesita arroparse de instrumentos que agranden y disminuyan los problemas al igual que lo hacen las lentes.

Así define la experiencia como un producto, un artefacto que se construye en el intento por denunciar y cambiar una situación social de opresión. Las condiciones de la opresión marcan un topos que debe ser recogido para articular un nuevo espacio de resignificación, un nuevo espacio político. Para Haraway la experiencia de las mujeres es un lugar de resignificación, un aparato de producción de nuevas significaciones mediante la lógica de una mirada diferente. Es un espacio por construir y el cíborg es la metáfora óptica que le permite justamente pensar ese espacio sin géneros, sin sexualidad. Por lo tanto, podemos decir que 
se convierte en un espacio de resignificación. El cíborg, además, como metáfora visual que es logra desplazar los intentos de la experiencia de las mujeres entendida en el sentido más clásico de reconstrucción del agente social, huyendo de los límites que las identidades imponen. El cíborg se define como un momentoposición para mirar y para actuar.

La relación entre estas dos nociones queda más clara en palabras de la propia autora:

Los movimientos internacionales feministas construyeron la «experiencia de las mujeres» y, asimismo, destaparon o descubrieron este objeto colectivo crucial. Tal experiencia es una ficción y un hecho político de gran importancia. La liberación se basa en la construcción de la conciencia de la comprensión imaginaria de la opresión y, también, de lo posible. El cíborg es materia de ficción y experiencia viva que cambia lo que importa como experiencia de las mujeres a finales de este siglo (Haraway, 1995, 253).

La autora afirma que el cíborg es nuestra ontología porque lo que somos es momentos-posición de conocimiento e intervención en una sociedad-red. Pero al mismo tiempo nos otorga nuestra política porque no es una posición inocente, sino que siempre es una localización interesada que reclama la instancia política como conformadora de la posición donde está. La política no solamente engulle la epistemología; toma además las riendas de la demarcación ontológica. Lo que son las mujeres depende de la posición que adopten en una compleja red de relaciones semiótico-materiales que es nuestra sociedad. El agente social y el agente epistemológico son lo mismo, dependen de la capacidad para superar las significaciones dadas en un proceso de semiosis ilimitada. De este modo, el conocimiento es un acto de responsabilidad porque es un acto político, la responsabilidad está en la posición que se adopte para enfrentar los problemas.

Por lo tanto, la experiencia de las mujeres vincula unos cuerpos sexuados en femenino al referirlos a una localización compartida en un proceso de atribución de significaciones. Las posiciones cíborg, por un lado, permiten detectar los problemas de la semiosis-material, la tecnociencia, en la que las mujeres están inmersas -localizadas- $y$, por otro, son las promotoras de cambios en ese proceso semiótico-material mediante lo que Haraway denomina política de la articulación.

Haraway entiende el conocimiento en clave política, o mejor dicho, establece una hábil política del conocimiento donde la localización tiene un papel central: «Lucho a favor de políticas o de epistemologías de la localización, del posicionamiento y de la situación, en las que la parcialidad y no la universalidad es la condición para que sean oídas las pretensiones de lograr un conocimiento racional» $(1995,335)$. La localización permite elaborar mapas de conciencia, concebir su propuesta como una cartografía política. Haraway propone diferentes posibilidades de localización que, como puntos de un mapa, orientan las reconfiguración del agente social. Sin caer en los fetichismos de la representación desde la que se pueden comprender los trabajos cartográficos, Haraway propone 
viajes de expedición por los mares de la materialidad y semioticidad de un espacio y un tiempo que se entrelazan con la propia historicidad del ojo expedicionario.

La cartografía política para Haraway es una forma de buscar nuevas significaciones, que según una de las autoras clásicas de la teoría feminista, Teresa de Lauretis, sería el cometido de la experiencia de las mujeres. La localización de las mujeres en el cambio de siglo, con la renuncia a las identidades sustantivas, no priva a ésta de capacidad para actuar. Es necesario redefinir el quehacer político para que las mujeres sean capaces de intervenir. El sujeto mujeres es una localización en una red que conoce y actúa, sin olvidar que es una materialidadsemiótica y que vive una historicidad que no ha escogido y que, por tanto, como señalaba Beauvoir, es fruto de la situación y de la elección.

Por lo tanto, la situación deviene una noción clave para entender el sujeto mujeres en todas sus articulaciones, tanto políticas como cognitivas. Las mujeres son parte de un movimiento fluido e historizado y se construyen en una red de elementos que se implican entre sí. Por lo tanto, la posición en la que las mujeres se encuentran puede ser empleada activamente como una localización para la construcción de significados, un lugar desde donde el significado se construye, más que un simple lugar desde donde el significado puede ser descubierto. Esta reflexión es fundamental para analizar el papel de las mujeres en la trayectoria de las políticas públicas de igualdad en los últimos veinte años. Lo fundamental en estas políticas, al igual que en las metáforas de Haraway, estriba en cambiar los procesos de atribución de significados. Agregar la experiencia de las mujeres cambia la receta.

\section{NUEVAS RECETAS PARA LAS POLÍTICAS DE IGUALDAD: IGUALDAD DE OPORTUNIDADES, ACCIÓN POSITIVA Y MAINSTREAMING}

El diseño de las políticas públicas de igualdad parte de la comprensión de las desigualdades de género que estructuran nuestra sociedad como el producto de un sistema dicotómico y jerárquico que atribuye distintos trabajos, espacios, recursos y poder a los hombres y a las mujeres, sistema que se reproduce de forma colectiva e individual. Los hombres y las mujeres no tienen la misma presencia en el ámbito público ni se valoran por igual sus opiniones y sus acciones. Tampoco acceden por igual a los recursos ni gozan de la misma posición en los espacios de toma de decisiones. El orden simbólico donde viven y se piensan hombres y mujeres subordina lo femenino a lo masculino en un sentido amplio. Esa compleja maraña de relaciones y valoraciones está presente en todos los planos de la vida pública y privada y, de este modo, ha empapado el quehacer político durante siglos.

Las políticas de igualdad vienen a modificar la situación dada introduciendo la perspectiva de género en el diseño y planificación de las políticas públicas. Su 
objectivo básico es detectar la discriminación y establecer los cambios estructurales que la puedan eliminar. Son, sin lugar a dudas, deudoras de las reivindicaciones y teorizaciones del movimiento feminista, y, por lo tanto, también de la mayor importancia otorgada a unos temas sobre otros. Sin embargo, estamos ya en un momento que nos permite cierta perspectiva sobre la implementación y resultado de las políticas de igualdad. Podemos afirmar que existe una evolución de las mismas que se corresponde con tres enfoques políticos: las políticas públicas basadas en la igualdad de oportunidades, la apuesta por la acción positiva y, más recientemente, la primacía del mainstreaming (Astelarra, 2005, 74).

En la actualidad existe un consenso generalizado para adoptar como estrategia política lo que se conoce como mainstreaming de género. El mainstreaming de género fue asumido por la Plataforma para la Acción de la Cuarta Conferencia Mundial sobre Mujeres de Naciones Unidas que se celebró en 1995. Este concepto, traducido por transversalidad, supone realmente un cambio respecto a las políticas de igualdad entendidas como «igualdad de oportunidades» y «acción positiva».

La igualdad de oportunidades se ha definido como el proceso de cambio legislativo que permite a todos los individuos tener las mismas oportunidades. Es cierto que para las mujeres éste fue un paso decisivo. El reconocimiento de la igualdad ante la ley permitió en un primer momento el reconocimiento formal de derechos y el acceso igualitario de las mujeres a la educación. El feminismo ilustrado apostó en sus inicios por esta línea de trabajo, lo que supuso hacer de la conquista de los derechos políticos y del acceso a la educación su bandera. La igualdad de oportunidades, inscrita en la tradición liberal, tiene sus limitaciones claras. La fundamental es olvidar que el espacio privado organiza la vida de hombres y mujeres de una forma discriminatoria para estas últimas. El rol de cuidadoras de las mujeres organiza la sociedad de tal forma que la igualdad de oportunidades en el espacio público no se garantiza sólo a través de la regulación del mismo.

Podemos realizar un paralelismo entre la igualdad de oportunidades y el empirismo feminista. En los dos casos se entiende necesaria una modificación normativa o cognitiva para dar cabida a los sujetos excluídos. Sería algo parecido a introducir parches correctores por un lado en el corpus legislativo de los propios estados y por otro en el propio método científico a través del reconocimiento de los valores contextuales como valores epistémicos (Longino, 1990). No es objeto de este artículo profundizar en esta idea pero consideramos que este paralelismo puede explicar parte de las dificultades para conseguir un punto de partida igual para acceder al ámbito público por parte de hombres y mujeres.

Las limitaciones a las que se hacía referencia en el párrafo anterior llevaron a modificar la estrategia de la igualdad de oportunidades hacia la acción positiva. Desde la óptica de ésta la intervención política debe corregir la desventaja de las mujeres en el mundo público a través de acciones concretas que las primen. Entre los ejemplos más típicos destaca el sistemas de cuotas, que ha propiciado no sólo la entrada de las mujeres en la política sino también ha contribuido a su decisivo empoderamiento; asimismo es significativo a este respecto las líneas de 
subvención específicas para la contratación de mujeres o los programas especiales para el emprendimiento femenino. En ambos casos los resultados se han valorado muy en positivo y han marcado un importante avance tanto en el ámbito de la democracia paritaria como en el aumento de la tasa de actividad femenina. Como podemos ver la acción positiva se ha movido siempre en la lógica de primar la entrada de las mujeres en el ámbito público como estrategia para finalizar la discriminación. En este punto, permítaseme recordar que la propia Simone de Beauvoir apostó por el trabajo remunerado como única fórmula para la liberación en el capítulo final de El segundo sexo.

Es necesario reconocer el papel trascendental de la acción positiva en las políticas de igualdad, pero también debemos mostrar la incapacidad que ha demostrado para transformar el espacio privado y modificar el rol de cuidadora de las mujeres. Como señala Astelarra:

Ellas siguen siendo las responsables, en forma total o parcial, del ámbito familiar y doméstico, independientemente de cualquier otra actividad familiar que desempeñen, y la doble jornada se convierte en su modo de vida. La evaluación de la implementación de las políticas de igualdad de oportunidades y de acción positiva conduce inevitablemente al ámbito privado y su incidencia en las actividades de las mujeres. Aún cuando modifican la situación de las mujeres, subyace el problema de cómo transformar la organización social que sustenta la discriminación y el rol que ocupan en la familia. (Astelarra, 2005, 79).

El rol de cuidadoras que ocupan las mujeres en la familia y su mayor cercanía a la naturaleza y a los procesos materiales de la vida ha sido uno de los temas destacados de las reflexiones de las epistemólogas. Especial relevancia cobra este tema en la teoría del punto de vista feminista. Autoras como Nancy Hartsock o Evelyn Fox Keller han establecido toda una línea argumental sobre el papel determinante que tiene la educación diferenciada en la configuración de la racionalidad abstracta masculina y la capacidad más relacional que desarrollan las mujeres. Muchas de estas apreciaciones se han asumido por parte de discursos sobre el liderazgo en el mundo de la empresa y la política con un planteamiento más esencialista y menos crítico con los elementos culturales.

El análisis de la incidencia de la acción positiva ha señalado la necesidad de tener presente la experiencia de las mujeres y su situación en el entramado social para poder diseñar políticas públicas que efectivamente signifiquen una transformación total del sistema de dominación patriarcal. En la clave que asumimos el pensamiento de Haraway la acción positiva conceptualiza la situación de las mujeres como una herencia y una elección que éstas deben modificar en un proceso de resignificación constante. Por resignificar entendemos en este ámbito cambiar la propia estructura social que modifica la asignación de papeles. Incluir mujeres ha significado también introducir en la receta su perspectiva y su capacidad para modificar las reglas dadas, ha significado plantearse la cuestión del ámbito de la vida. 
Las conclusiones sobre la acción positiva son muy importantes para interpretar el género como un sistema de relaciones. La entrada de las mujeres en el espacio público no modifica por sí misma todo un entramado de relaciones que mantienen la vida. Poner en la agenda política esta cuestión es un logro importante. El debate sobre la conciliación no ha hecho más que comenzar y ya se ha convertido en una preocupación política de primera magnitud porque muestra algo que las feministas llevaban décadas señalando, a saber, que detrás del contrato social existe uno previo de carácter sexual (Pateman, 1995). Y esto gracias a una escenificación de la experiencia de las mujeres y una comprensión de la conciliación como un problema no de las mujeres sino como una preocupación de toda la sociedad que implica una transformación estructural.

En este sentido la estrategia de mainstreaming pretende analizar el impacto diferencial de género que tienen las actuaciones públicas con el objetivo de conseguir el tan ansiado cambio estructural. Tomemos como definición la que utiliza el Consejo de Europa:

El mainstreaming de género es la organización (la reorganización), la mejora, el desarrollo y la evaluación de los procesos políticos, de modo que una perspectiva de igualdad de género se incorpore en todas las políticas, a todos los niveles y en todas las etapas, por los actores normalmente involucrados en la adopción de medidas prácticas (Consejo de Europa, 1999).

Como podemos observar el mainstreaming pretende introducir la perspectiva de género desde el inicio del diseño de las políticas hasta su evaluación última. Más allá de añadir «mujeres», el objetivo es cambiar la receta. En este aspecto es muy importante tomar nota de lo que fueron las políticas públicas para el desarrollo y, como analiza Sandra Harding, ser conscientes de que «la incorporación de las mujeres en el desarrollo de la ciencia y la tecnología ha significado la incorporación de unas pocas mujeres de élite a las áreas de alto prestigio —un Premio Nobel aquí, un puesto universitario allá — o, por otro lado, el reclutamiento del trabajo de las mujeres para trabajos poco especializados y mal pagados que benefician a sus familias, a los empleados, a los gobiernos locales, a los consumidores del Norte y a las corporaciones multinacionales, pero no a las propias mujeres» (Harding, 1986, 297).

El mainstreaming sitúa la responsabilidad en todos los actores implicados, pero su aplicación requiere de una correcta comprensión del concepto (Lombardo y Meier, 2006). No basta con introducir dos o tres accciones concretas en cada departamento administrativo sino que se necesita una transformación sustancial en la estructura del trabajo institucional y burocrático. La primera exigencia es definir claramente en qué consiste la igualdad de género y determinar los objetivos a conseguir. En segundo lugar, la estrategia de transversalidad requiere de una planificación coordinada interdepartamental, lo que implica contar con personal formado en género de todas las áreas en las que se va a trabajar y, por último, ser capaces de evaluar los resultados contando con indicadores claros de género. 
En el desarrollo de estos tres pasos el contexto es claramente determinante, es decir, los objetivos concretos en el diseño de una determinada política pública deben partir de un conocimiento de la propia realidad y de la situación que viven las mujeres en función de las relaciones de género predominantes. Además no podemos olvidar que el conocimiento de esta situación supone contar con ellas como interlocutoras: es realmente difícil creer en un proceso transformador si no valoramos las posibilidades de participación e intervención de las propias mujeres.

Es en este punto donde las reflexiones epistemológicas pueden aportan un marco interpretativo provechoso. El mainstreaming no puede ser sólo un instrumento. Debemos cargarlo de una lógica feminista que contemple el contexto, la situación de las mujeres y la diversidad de desigualdades con las que intersecciona el género. En este momento encontramos variados estudios y escritos que destacan las diferentes formas de aplicar la transversalidad en los países de la Unión Europea (Walby, 2005a). En ellos se refleja la necesidad de establecer unos objetivos comunes, pero que al mismo tiempo tengan presente el contexto en el que se aplican las políticas públicas.

Podemos afirmar que como denominador común de las políticas europeas de mainstreming se establece un concepto amplio de igualdad de género que tiene por objetivo acabar con las causas interconectadas que crean la relación asimétrica entre los sexos en áreas como la familia, el trabajo remunerado, la política, la sexualidad, la educación, la cultura o la violencia machista. Esto muestra, por otro lado, la necesidad de centrarse siempre en la aplicación del mainstreaming en las relaciones de género. Un ejemplo muy clarificador al respecto es el siguiente: la Unión Europea ha centrado las políticas de familias fundamentalmente en las mujeres mientras ha olvidado la inclusión de los hombres (Lombardo y Meier, 2006, 157) y las relaciones de género. Así el problema de la conciliación de la vida familiar, laboral y personal siempre aparece vinculado a las mujeres y como un tema directamente relacionado con el déficit demográfico y los cambios en los estilos de vida familiar. El marco interpretativo de la igualdad de género que sustenta esta planificación política se basa en la organización del trabajo y deja al margen la organización de la intimidad (Lombardo, 2003). Si no tenemos presente las relaciones entre los géneros en la estructuración social no podremos analizar el asimétrico uso de los tiempos que viven en su cotidianidad hombres y mujeres.

Como señala Lombardo los propios documentos sobre conciliación son una limitación de la igualdad de oportunidades ya que reproducen y consolidan el rol de las mujeres como cuidadoras principales. Por lo tanto, el mainstreaming no se aplica adecuadamente y es necesario introducir en la estrategia con que se lo concibe contenido feminista que desmonte la idea de centrar la organización social sólo en el trabajo remunerado y tome la reproducción de la vida —o ámbito privado - como un tema social que necesita estar presente en la agenda política por sí mismo. Dejemos de hablar en términos de conciliación de las mujeres y comencemos a hablar de corresponsablidad de todos los actores implicados en las tareas de cuidado (Xunta de Galicia, 2006). 
Asumir o no la primacía política del trabajo remunerado es un aspecto fundamental para definir la igualdad de género. Tal es así que para algunas feministas dicho trabajo no puede constituir una ruta universal y contingente hacia la que avanzan las mujeres, sino que existen otras variables para medir el desarrollo de las mujeres. El reto estriba en establecer indicadores de género más allá del empleo y los campos relacionados con el mismo —un ejemplo es la violencia contra las mujeres-. Sólo cuando tengamos establecidos indicadores de género que excedan el ámbito público podremos medir el avance de las mujeres (Walby, 2005a).

El diseño de los indicadores es vital para poder evaluar la incidencia de las políticas de igualdad. Es necesario que la perspectiva feminista esté presente en la creación del conocimiento en diferentes áreas, como es el caso de la estadística. Las epistemólogas ya lo han afirmado en reiteradas ocasiones: no necesitamos un conocimiento feminista sino construir el conocimiento como feministas (Adán, 2006). Es justamente en ese punto donde las políticas públicas necesitan de la epistemología a fin de contar con un buen diagnóstico de la situación de partida que permita una planificación basada en una estrategia conjunta de mainstreaming pero que se rellene con un contenido feminista y se pueda medir con unos indicadores fiables para el avance de las mujeres.

Los objetivos del milenio marcan claramente la necesidad de poder evaluar estos avances. Muchas han sido las críticas a la aplicación de estos objetivos por no tener presente el contexto (Walby, 2005b), además de por abusar del mainstreaming en un sentido meramente burocrático. Debemos tener presente siempre las vidas situadas de las mujeres en cada punto del planeta para evitar que las políticas de igualdad se conviertan en un movimiento de papeles a nivel administrativo y olviden, en palabras de Haraway, que los procesos de resignificación tambien están cargados de materialidad.

\section{SIN CONCLUSIONES: PARA SEGUIR COCINANDO NUEVAS RECETAS}

La cocina permite que el salón esté limpio y ordenado, la cocina permite que los tiempos del salón sean de debate y diversión, la cocina permite que el ocio y el descanso reine en otros espacios de la casa. La casa existe porque la organización de la cocina es invisible. Cuando la cocina reivindica su espacio y su tiempo la casa cambia, lo periférico es central y lo central se desdibuja. Las relaciones de colaboración de la cocina y sus conocimientos prácticos y cotidianos adquieren el valor que se merecen. Las mujeres contamos ya con una tradición en el arte de transformar lo periférico y lo central, los márgenes y el centro.

La teoría feminista ha sido capaz de transfomar saberes y discursos así como prácticas y significados. Las políticas de igualdad han contribuido enormemente al desarrollo democrático de las sociedades actuales. Han redefinido la situación de la cocina, han ensayado recetas nuevas buscando ingredientes ca- 
paces de subvertir las relaciones de género. Seguro que en la despensa tenemos artillería teórica y práctica para avanzar; la dificultad reside en combinar los ingredientes que tenemos, y que nos han sido útiles, con otros nuevos. Debemos planificar según el contexto - local, comarcal, nacional, estatal, europeo, mundial- las políticas más adecuadas a los objetivos que perseguimos. El contexto es determinante y el buen conocimiento del mismo también. No es suficiente con establecer procedimientos administrativos para implementar las políticas de igualdad. Tenemos que ir un paso más allá y modificar los propios marcos interpretativos en los que nos movemos. La comunidad epistémica feminista tiene un reto importante: construir conocimientos que sirvan de base para diseñar y evaluar políticas de igualdad acordes con la situación que viven las mujeres y con el objetivo de transformar efectivamente las relaciones de género.

\section{BIBLIOGRAFÍA}

AdÁn, C. (2006): Feminismo y Conocimiento, A Coruña, Espiral Maior.

Amorós, C. (ed.) (2000): Feminismo y Filosofía, Madrid, Síntesis.

Astelarra, J. (2005): Veinte años de políticas de igualdad, Madrid, Cátedra.

Beauvoir, S. (1999): El segundo sexo, Madrid, Cátedra.

CONSEJO DE EUROPA (1999): Mainstreaming de género. Marco conceptual, metodología y presentación de «buenas prácticas». Informe final de las actividades del Grupo de especialistas en mainstreaming ( $E G-S-M S)$, Instituto de la Mujer, Ministerio de Trabajo y Asuntos Sociales, Serie documentos, n. ${ }^{\circ} 28$, Madrid.

LOMBARDO, E. (2003): «El mainstreaming de género en la Unión Europea», Aequalitas. Revista Jurídica de Igualdad de Oportunidades entre Mujeres y Hombres, vol. 10-15, pp. 6-11

LOMBARDO, E. y MeIER, P. (2006): «Gender Mainstreaming in the EU. Incorporating a Feminist Reading?», European Journal of Women's Studies, vol. 13 (2), pp. 151-166, Londres, SAGE Publications.

Longino, H. (1990): Science as Social Knowledge, N. J., Princenton University Press.

HARAWAY, D. (1995): Ciencia, Cyborg y mujeres. La reinvención de la naturaleza, Madrid, Cátedra.

Harding, S. (1986): The Science Question in Feminism, Ithaca, Cornell University Press.

Pateman, C. (1995): El contrato sexual, Barcelona, Anthropos.

Rose, H. (1994): Love, Power and Knowledge, Cambridge, Polity Press.

GAYLE Rubin, «The Traffic in Women: Notes in the "Political Economy" of Sex», en Rayna R. Reiter (ed.), Toward and Anthropology of Women, Nueva York y Londres: Monthly Review Press, 1975, pp. 157-210.

Xunta de Galicia (2006): Plan de fomento da corresponsabilidade, Santiago de Compostela, Secretaría Xeral da Igualdade, Xunta de Galicia.

WALBY, S. (2005a): «Measuring women's progress in a global era», Unesco 2005, Oxford, Blackewell, pp. 371-387.

WALBY, S. (2005b): «Comparative Gender Mainstreaming in a Global era», International Feminist Journal of Politics, 7:4, pp. 453-470. 


\section{RESUMEN}

Desde finales de los años noventa del pasado siglo, cuando en España se tomó conciencia sobre la gravedad del fenómeno de la violencia de género, las Administraciones Públicas, empujadas por el feminismo, han ido afinando las herramientas conceptuales y estadísticas a fin de lograr la contabilidad imprescindible para la intervención pública. A partir de lo que se incluya bajo esta rúbrica se ha de distinguir entre «la violencia de género» $\mathrm{y}$ «las cifras de la violencia»: entre la definición «abstracta» de la violencia y las cifras median los «indicadores» de qué se considera violencia, y ello depende en buena parte, en primer lugar, de la toma de conciencia del problema y, en segundo lugar, de los diversos intereses en liza; en definitiva, es una cuestión política. En el presente artículo analizaremos las razones de sucesivas ampliaciones de las cifras de la violencia y revisaremos críticamente algunas de las transformaciones conceptuales que las han acompañado.

\section{PALABRAS CLAVE}

Género, experiencia de las mujeres, situación, políticas de igualdad, mainstreaming.

\section{ABSTRACT}

In feminist theory, the epistemological debate has happened in a peripheral way. It has been built up as a second order reflection that has strengthened partially the feminist knowledge leaving aside its likely political dimension. In the same way, in the design and implementation of equal politics epistemological issues were dealt in a partial way. However, to understand nowadays equal politics is central to feminists how and where from knowledge is being created. The epistemological feminist community has to focus on studies, indicators and statistics to improve women's life and to eliminate the structures that reproduce gender inequalities. In this article we consider the need of reviewing from an epistemological point of view some notions like gender, women's experience and situation. These are key issues to understand the evolution of equal politics in the last twenty years. They are useful as well to think about the advantages and disadvantages of a tool such as mainstreaming.

\section{KEY WORDS}

Gender, women's experience, situation, equality politics, mainstreaming. 